\title{
A closed-form solution for energy-efficiency optimization in multi-user downlink NOMA
}

\author{
Hajar El Hassani*, Anne Savard ${ }^{\dagger}$ and E. Veronica Belmega* \\ *ETIS UMR 8051, CY Cergy Paris Université, ENSEA, CNRS, \\ F-95000, Cergy, France \\ ${ }^{\dagger}$ IMT Lille Douai, Univ. Lille, CNRS, UMR 8520 - IEMN, \\ F-59000 Lille, France \\ Email: *hajar.el-hassani@ensea.fr, ${ }^{\dagger}$ anne.savard@imt-lille-douai.fr, *belmega@ensea.fr
}

\begin{abstract}
This paper investigates the energy efficiency of a multi-user downlink non-orthogonal multiple access (NOMA) system. The energy-efficiency maximization representing the tradeof between the sum rate versus the overall power consumption is formulated as a bi-criterion optimization problem; being convex, scalarization is suitable for its solution. Assuming that each individual user has a minimum quality of service constraint, we prove that the solution of the scalarized objective can be obtained in closed form. Hence, our solution characterizes the entire Pareto-optimal boundary of the rate vs. power tradeoffs. In the special case of maximizing the ratio sum rate vs. overall power, our solution reduces the complexity of the Dinkelbach procedure to a univariate bisection method. At last, our simulation results highlight the benefit of NOMA over OMA in terms of the rate vs. power optimal tradeoff.
\end{abstract}

Index Terms-NOMA, energy efficiency, bi-criterion optimization, minimum QoS constraints

\section{INTRODUCTION}

Non-orthogonal multiple access (NOMA), which enables multiple users to communicate over the same frequency, time or spreading-code resources, has been identified as a very promising technology to improve the spectrum efficiency of future communication networks [1]-[4]. Furthermore, it was shown that NOMA outperforms OMA in terms of device connections and spectrum efficiency, making it very suitable for Internet of Things (IoT) [5] applications. Nevertheless, energy consumption remains a major issue and requires to allocate the network resources in an efficient manner. In the last couple of years, resource allocation problems for NOMAbased systems have been widely studied in the literature from an energy-efficient perspective. Both uplink [6] and downlink [7], [8] scenarios have been studied given the recent advances in user equipment making possible the implementation of successive interference cancellation (SIC) in practice [9], [10].

In this paper, we study the energy efficiency of a multiuser NOMA system, formulated as a bi-criterion convex optimization problem, similarly to [8]. Aside from the power budget constraint, we also consider minimum QoS constraints

This work has been supported by the ELIOT ANR-18-CE40-0030 and FAPESP 2018/12579-7 project and IRCICA, CNRS USR 3380, Lille, France.

978-1-7281-4490-0/20/\$31.00 @ 2020 IEEE for all links. Remarkably, we provide the closed-form solution - known only in the special case of two users [3] - for the general case of $K \geq 2$ users, as opposed to [6]-[8] (in which only iterative methods were proposed). Our closed form solution allows us to fully characterize the entire Paretooptimal boundary of the rate vs. power tradeoff points. Our numerical simulations show that NOMA outperforms OMA both in terms of achievable sum rate (up to $9.4 \%$ gain NOMA vs. OMA) and of consumed power (up to $33 \%$ excess OMA vs. NOMA), irrespective from the tradeoff parameter.

The closest works to ours are [11] and [12], in which different closed-form power allocation solutions have been provided in multi-user downlink NOMA systems. In [11], the rate maximization problem was considered, in which the transmission always takes place at maximum power (which is not power-efficient). In [12], the energy efficiency defined as the ratio between the sum rate and the overall power consumption was studied. By using a skillful variable change, which lead to an intermediary closed-form solution to a multi-variate non-convex problem, finding the maximum energy efficiency in a multi-user setting was reduced to a univariate bisection method. Nevertheless, the solutions in [11], [12] provide only very specific points on the Pareto-optimal boundary, whereas our closed-form solution provides all rate vs. power optimal tradeoffs.

\section{SYSTEM MODEL AND PROBLEM FORMULATION}

The network under study is composed of a single transmitter (e.g., base-station, IoT access point, etc.) and $K$ receivers. The transmitter employs a superposed coding technique and the received signal at receiver $k$ writes as

$$
Y_{k}=h_{k} X+Z_{k}=h_{k} \sum_{i=1}^{K} X_{i}+Z_{k}
$$

where $X_{i}$, of average power $p_{i} \geq 0$, denotes the message intended for receiver $i$ and $Z_{k}$, of variance $\sigma_{k}^{2}$, is the additive white Gaussian noise at receiver $k$.

We assume that the channels are ordered as follows

$$
\left|h_{1}\right|^{2} / \sigma_{1}^{2}>\left|h_{2}\right|^{2} / \sigma_{2}^{2} \geq\left|h_{3}\right|^{2} / \sigma_{3}^{2} \geq \ldots \geq\left|h_{K}\right|^{2} / \sigma_{K}^{2},
$$


which for a NOMA system means that the $k$-th strongest user with respect to the channel quality decodes first the signals of the weaker users $j \geq k+1$ using SIC, and only sees the interference coming from the stronger users $j \leq k-1$. Hence, the achievable rate of user $k$-th writes as [11]

$$
R_{k}^{\mathrm{NOMA}}(\mathbf{p})=\log _{2}\left(1+\frac{\left|h_{k}\right|^{2} p_{k}}{\left|h_{k}\right|^{2}\left(p_{1}+\ldots+p_{k-1}\right)+\sigma_{k}^{2}}\right),
$$

where $\mathbf{p}=\left(p_{1}, \ldots, p_{K}\right)$ denotes the power allocation vector.

The network is constrained by an overall power budget at the transmitter such that $\sum_{i} p_{i} \leq P_{\max }$ as well as by individual minimum QoS constraints given as $R_{k}^{\mathrm{NOMA}}(\mathbf{p}) \geq R_{\min , k} \forall k$. These constraints define the set of feasible power allocation vectors given as

$$
\mathcal{P} \triangleq\left\{\mathbf{p} \in \mathbb{R}_{+}^{K} \mid \sum_{k=1}^{K} p_{k} \leq P_{\max }, R_{k}(\mathbf{p}) \geq R_{\min , k}, \forall k\right\} .
$$

Throughout the paper, we will use the following notations to simplify the mathematical derivations: $\Gamma_{k}=\frac{\left|h_{k}\right|^{2}}{\sigma_{k}^{2}}$,

$A_{k}=2^{R_{\min , k}}, \theta_{k}=\sum_{i=1}^{k} p_{i}, \forall k \geq 1$, and $\theta_{0}=0$. Using these notations, the feasible set can be expressed as a convex polytope (afine inequality constraints):

$$
\mathcal{P}=\left\{\mathbf{p} \in \mathbb{R}_{+}^{K} \mid \theta_{K} \leq P_{\max }, \theta_{k} \geq A_{k} \theta_{k-1}+\frac{A_{k}-1}{\Gamma_{k}}, \forall k\right\} .
$$

\section{A. Energy efficiency as a bi-criterion problem}

Energy efficiency measures the tradeoff between the overall throughput and the power consumption of the network and can be formulated mathematically as a bi-criterion optimization problem [13], which in our case writes as

$$
\max _{\mathbf{p} \in \mathcal{P}}\left(\sum_{k=1}^{K} R_{k}^{\mathrm{NOMA}}(\mathbf{p}) ;-\sum_{k=1}^{K} p_{k}-P_{c}\right),
$$

where $P_{c}$ denotes the constant circuit power consumption. Notice that the two above objectives are contradictory: to maximize the sum rate, the network transmissions take place at full power, which obviously is not energy-efficient; and to minimize the power consumption, there should be no transmission, which is neither throughput- nor QoS-efficient.

To solve such a bi-criterion optimization problem, we need to find the Pareto-boundary of the feasible set of the rate-power pairs. This boundary contains all the Pareto-optimal solutions, which represent the rate-power feasible pairs which cannot be improved in both objectives simultaneously while remaining in the feasible set. Under the channel order assumed, the sum rate is jointly concave w.r.t. $\mathbf{p}$ as shown in [11]. Hence, the optimization problem (2) is convex and finding the Paretoboundary reduces to maximizing the weighted sum of the two opposing objectives [14]:

$$
\max _{\mathbf{p} \in \mathcal{P}} \sum_{k=1}^{K} R_{k}^{\text {NOMA }}(\mathbf{p})-\alpha\left(\sum_{k=1}^{K} p_{k}+P_{c}\right),
$$

where $\alpha \geq 0$ is the parameter that trades off between rate and power consumption and sweeps the entire Pareto-boundary.
It can also be interpreted as a measure of the unit power consumption cost.

In what follows, we first provide the closed-form solution and then, we show how to exploit this solution in the maximization of the ratio between the sum rate and the overall power consumption.

\section{ENERGY EFFICIENCY FOR DOWNLINK NOMA}

Because of the QoS constraints, the feasible set may be void. Indeed, depending on the channel conditions, the QoS requirements for all users may not be met under the available power budget. Necessary and sufficient feasibility conditions were introduced in [11].

Proposition 1 ([11]). The optimization problem (2) is feasible if and only if the following condition on the system parameters holds: $P_{\max } \geq P_{\min } \triangleq \sum_{i=1}^{K} \frac{A_{i}-1}{\Gamma_{i}} \prod_{j=i+1}^{K} A_{j}$.

Intuitively, $P_{\min }$ denotes the overall minimum power required for all the QoS constraints to be met with equality.

\section{A. Closed-form solution}

Exploiting the notations $\Gamma_{k}$ and $\theta_{k}$, the optimization problem under study can be rewritten as

$$
\begin{array}{ll}
\max _{\mathbf{p}} & \sum_{k=1}^{K} \log _{2}\left(\frac{1+\Gamma_{k} \theta_{k}}{1+\Gamma_{k} \theta_{k-1}}\right)-\alpha\left(\theta_{K}+P_{c}\right) \\
\text { s.t. } & \theta_{K} \leq P_{\max }, \\
& \theta_{k} \geq A_{k} \theta_{k-1}+\frac{\left(A_{k}-1\right)}{\Gamma_{k}}, \forall k \in\{1, \ldots, K\},
\end{array}
$$

which is a convex problem that we solve analytically and in closed-form using the Karush-Kuhn-Tucker (KKT) optimality conditions. Intuitively, under the assumed order of the channels, we can show that all users except for the strongest one (user 1) should meet their QoS with equality in order to maximize the overall energy efficiency.

Theorem 1. The optimal power allocation maximizing the energy efficiency in (2) for a downlink multi-user NOMA system is obtained in closed-form as follows:

$$
\begin{aligned}
p_{k}^{*}(\alpha)= & \left(A_{k}-1\right)\left(\frac{1}{\Gamma_{k}}+p_{1}^{*}(\alpha) \prod_{i=2}^{k-1} A_{i}\right. \\
& \left.+\sum_{i=2}^{k-1} \frac{A_{i}-1}{\Gamma_{i}} \prod_{j=i+1}^{k-1} A_{j}\right), \quad \forall k \geq 2, \\
p_{1}^{*}(\alpha)= & \min \left(\max \left(\overline{p_{1}}(\alpha) ; \frac{A_{1}-1}{\Gamma_{1}}\right) ; U_{1}\right),
\end{aligned}
$$

where $U_{1}$ and $\overline{p_{1}}(\alpha)$ are expressed below

$$
\begin{aligned}
U_{1} & =\frac{1}{\prod_{i=2}^{K} A_{i}}\left(P_{\max }-P_{\min }+\frac{A_{1}-1}{\Gamma_{1}} \prod_{j=2}^{K} A_{j}\right), \\
\overline{p_{1}}(\alpha) & =\frac{1}{(\ln 2) \alpha \prod_{i=2}^{K} A_{i}}-\frac{1}{\Gamma_{1}} .
\end{aligned}
$$


Proof. Let $\mathcal{L}$ be the Lagrangian of the convex problem (4):

$$
\begin{aligned}
\mathcal{L}= & \sum_{i=1}^{K} \log _{2}\left(\frac{1+\Gamma_{i} \theta_{i}}{1+\Gamma_{i} \theta_{i-1}}\right)-\alpha\left(\theta_{K}+P_{c}\right)+\lambda\left(P_{\max }-\theta_{K}\right) \\
& +\sum_{i=1}^{K} \beta_{i}\left(\theta_{i}-A_{i} \theta_{i-1}-\frac{A_{i}-1}{\Gamma_{i}}\right),
\end{aligned}
$$

where $\lambda$ and $\beta=\left(\beta_{1}, \ldots, \beta_{K}\right)^{T}$ are the positive Lagrange multipliers for the overall power constraint and the $K$ QoS constraints, respectively. The KKT optimality conditions are necessary and sufficient for optimality and they imply that $\frac{\partial \mathcal{L}}{\partial p_{k}}=0, \forall k$, at the solution. Thus, we also have that the difference between two consecutive Lagrange derivatives equals zero, $\frac{\partial \mathcal{L}}{\partial p_{k+1}}-\frac{\partial \mathcal{L}}{\partial p_{k}}=0$, which leads to the following equations

$$
A_{k+1} \beta_{k+1}=\frac{1}{\ln 2}\left(\frac{\Gamma_{k}}{1+\Gamma_{k} \theta_{k}}-\frac{\Gamma_{k+1}}{1+\Gamma_{k+1} \theta_{k}}\right)+\beta_{k},
$$

for all $k \in\{1, \ldots, K-1\}$.

Now, exploiting the assumption that $\Gamma_{1}>\Gamma_{2}, \beta_{1} \geq 0$ and $A_{2}>0$, the above equation for $k=1$ implies that $\beta_{2}>0$. Since $\Gamma_{k} \geq \Gamma_{k+1}$ and $A_{k+1}>0$, for all $k \geq 2$, the fact that $\beta_{2}>0$ has a cascading effect in the above equations and leads to $\beta_{k+1}>0, \forall k \in\{2, \ldots, K-1\}$. Hence, we have that $\beta_{k}>0, \forall k \geq 2$. This means that all QoS constraints of all weaker users, $k \geq 2$, are active at the solution and they meet no more than their minimum rate requirement:

$$
\theta_{k}=A_{k} \theta_{k-1}+\frac{A_{k}-1}{\Gamma_{k}}, \forall k>1 \text {. }
$$

By induction, we can show that

$$
\theta_{k}=\theta_{1} \prod_{i=2}^{k} A_{i}+\sum_{i=2}^{k-1} \frac{A_{i}-1}{\Gamma_{i}} \prod_{j=i+1}^{k} A_{j}+\frac{A_{k}-1}{\Gamma_{k}}, \forall k>1 .
$$

Knowing that $p_{k}=\theta_{k}-\theta_{k-1}$, for all $k>1$, and that $\theta_{1}=p_{1}$, the above equations allow us to express all the powers allocated to the weaker users as functions of $p_{1}$, the power allocated to the strongest user as in (5). Hence, the multi-variable problem in (4) is reduced to the following single variable problem:

$$
\max _{p_{1}} f_{1}\left(p_{1}\right) \text { s.t. } \frac{A_{1}-1}{\Gamma_{1}} \leq p_{1} \leq U_{1},
$$

where the new objective function is

$$
\begin{aligned}
& f_{1}\left(p_{1}\right)=\log _{2}\left(1+\Gamma_{1} p_{1}\right)+\log _{2} \prod_{i=2}^{K} A_{i} \\
& -\alpha\left(p_{1} \prod_{i=2}^{K} A_{i}+\sum_{i=2}^{K-1} \frac{A_{i}-1}{\Gamma_{i}} \prod_{j=i+1}^{K} A_{j}+\frac{A_{K}-1}{\Gamma_{K}}+P_{c}\right)
\end{aligned}
$$

and the upper bound on $p_{1}$, coming from the maximum power constraint, is given in (6).

It is obvious that the objective function $f_{1}\left(p_{1}\right)$ is concave w.r.t. $p_{1}$, hence the optimal solution $p_{1}^{*}(\alpha)$ is either the critical point $\overline{p_{1}}(\alpha)$ in (7) canceling the first order derivative, or one of the borders of the new feasible set, concluding the proof.

\section{B. Ratio between the sum rate and overall power}

A popular energy-efficiency metric is defined as the ratio between the sum rate and the overall power consumption [7], [12], [13], [15] measured in bits per Joule of consumed energy

$$
\eta_{E E}(\mathbf{p})=\frac{\sum_{k} R_{k}(\mathbf{p})}{\sum_{k} p_{k}+P_{c}},
$$

whose solution also lies on the Pareto-boundary of the bicriterion problem (2).

In [12], the authors proposed a skillful variable change for the maximization of $\eta_{E E}(\mathbf{p})$ leading to a closed-form intermediary solution and reducing the Dinkelbach procedure to a univariate bisection method.

Although our closed-form solution solves a different (more general) problem, it can also be exploited for the same purpose. Using fractional programming [7], [15] and our closed-form solution in Theorem 1, simplifies the optimization of (10) to finding the fixed point of the following function

$$
F(\alpha)=\sum_{k=1}^{K} R_{k}^{\mathrm{NOMA}}\left(\mathbf{p}^{*}(\alpha)\right)-\alpha\left(\sum_{k=1}^{K} p_{k}^{*}(\alpha)+P_{c}\right),
$$

where $\mathbf{p}^{*}(\alpha)$ is given in (5). This can be done simply by using the bisection method.

\section{Simulation RESUlts}

In this section, we compare the performance of NOMA and OMA in terms of both achievable sum rate and power consumption in the following setup: $K=3$ users, $P_{\max }=$ $10 \mathrm{~W}$ available transmit power, $P_{c}=1 \mathrm{~W}$ circuit power consumption, $R_{\min , k}=2$ bps, $\forall k$, i.e., all users have the same QoS requirement. We also assume that the noise variances are such that $\sigma_{k}^{2}=1, \forall k$ and that the channel gains are such that $h_{k} \sim \mathcal{C} \mathcal{N}(0,5), \forall k$. The curves are averaged over $10^{5}$ independent channel realizations.

Under OMA, the transmitter serves the $K$ users by performing time sharing with equal time slots. Each user $k$ is allocated a power $q_{k} \in\left[0, P_{\max }\right]$ and can thus achieve the rate $R_{k}^{\mathrm{OMA}}(\mathbf{q})=\frac{1}{K} \log _{2}\left(1+\Gamma_{k} q_{k}\right)$. Note that under OMA, the total amount of consumed power writes as $\frac{1}{K} \sum_{k=1}^{K} q_{k}+P_{c}$. For a fair comparison with NOMA, the same individual QoS constraints $\left\{R_{\min , k}\right\}_{k}$ are imposed in addition to the power budget $P_{\max }$ to compute the optimal power allocation under OMA, denoted by $\mathbf{q}^{*}(\alpha)$.

In Fig. 1(a) and Fig. 1(b), we illustrate the relative sumrate gain, $G(\alpha)$, of NOMA vs. OMA and the relative power consumption excess, $E(\alpha)$, of OMA vs. NOMA respectively, as a function of $\alpha$, the parameter that trades off between rate and power consumption. To be precise, the two performance metrics $G(\alpha)$ and $E(\alpha)$ are defined as

$$
\begin{aligned}
& G(\alpha) \triangleq \frac{\sum_{k}\left[R_{k}^{\mathrm{NOMA}}\left(\mathbf{p}^{*}(\alpha)\right)-R_{k}^{\mathrm{OMA}}\left(\mathbf{q}^{*}(\alpha)\right)\right]}{\sum_{k} R_{k}^{\mathrm{OMA}}\left(\mathbf{q}^{*}(\alpha)\right)}, \\
& E(\alpha) \triangleq \frac{\sum_{k}\left[\frac{1}{K} q_{k}^{*}(\alpha)-p_{k}^{*}(\alpha)\right]}{\sum_{k} p_{k}^{*}(\alpha)+P_{c}}
\end{aligned}
$$




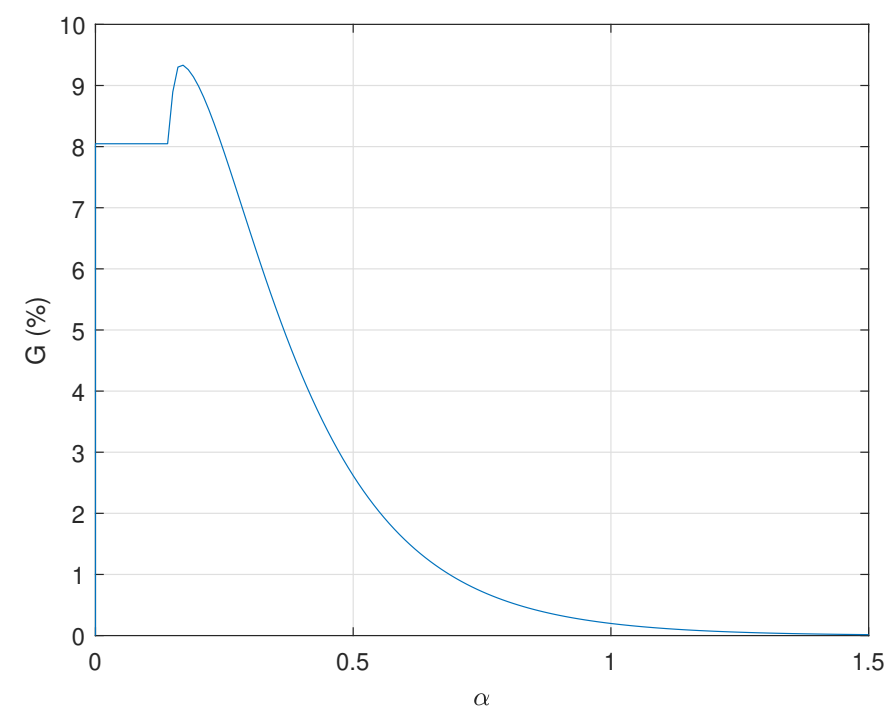

(a) Relative sum-rate gain of NOMA vs. OMA

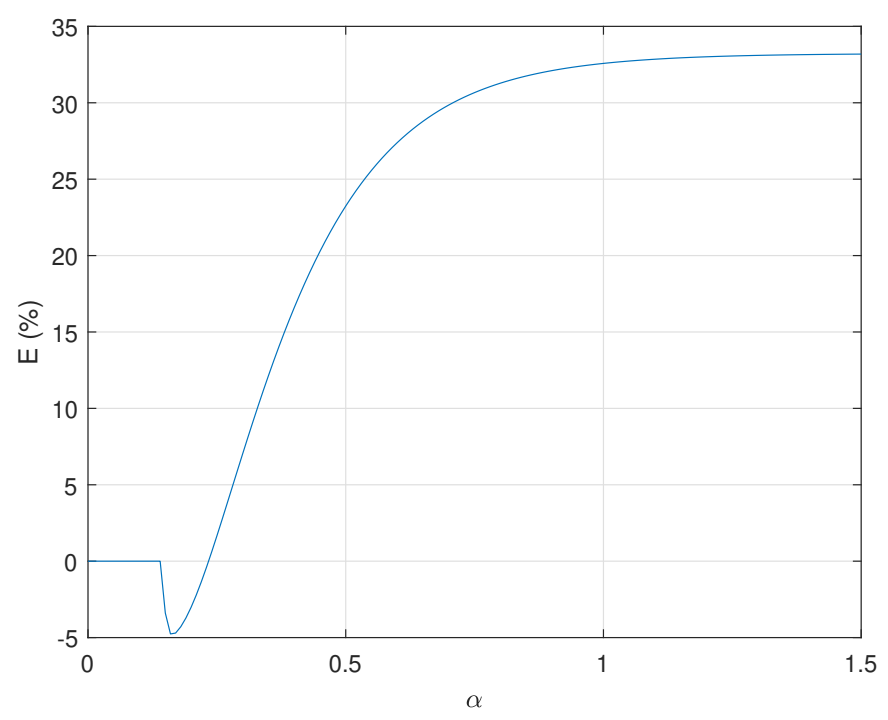

(b) Relative power consumption excess of OMA vs. NOMA

Fig. 1. NOMA vs. OMA as a function of the parameter $\alpha$ that tradeoffs between sum rate and power consumption in the energy-efficiency bi-objective optimization for $P_{\max }=10 \mathrm{~W}$. NOMA outperforms OMA in terms of sum rate for any $\alpha$. In terms of power consumption, for rate-driven objectives (small values of $\alpha$ ) OMA may consume less power (down to 5\%) than NOMA. At the opposite, for power-efficient objectives (large values of $\alpha$ ) OMA consumes more power (up to $34 \%$ ) than NOMA.

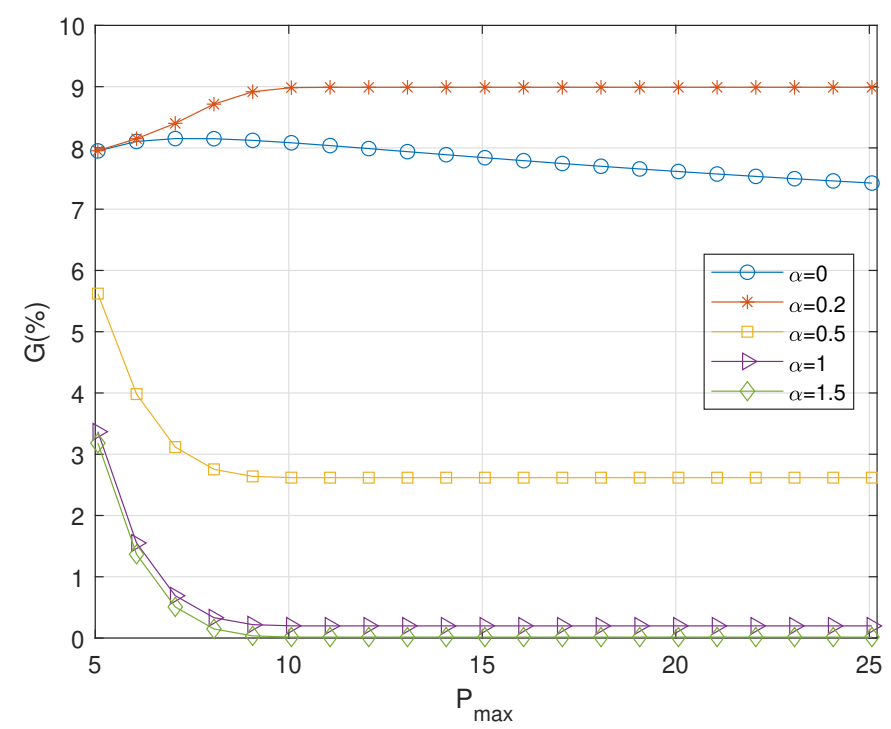

(a) Relative sum-rate gain of NOMA vs. OMA

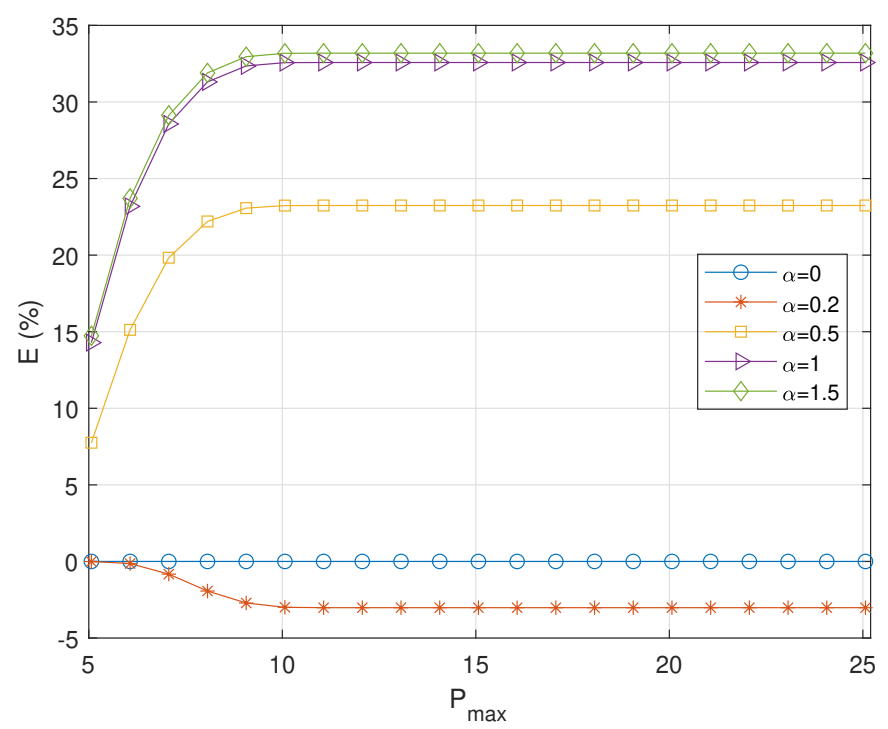

(b) Relative power consumption excess of OMA vs. NOMA

Fig. 2. NOMA vs. OMA as a function of the maximum power $P_{\max }$ for different values of $\alpha$. NOMA always performs at least as good as OMA in terms of sum rate and the gap between them decreases with $\alpha$. In terms of power consumption, for rate-driven objectives (small values of $\alpha$ ) OMA may consume less power (down to 4\%) than NOMA. At the opposite, for power-efficient objectives (large values of $\alpha$ ) OMA consumes more power (up to 34\%) than NOMA.

In Fig. 1, notice that the gain $G$ is always non-negative, which means that NOMA cannot decrease the achievable sum rate compared to OMA and, for low values of $\alpha$ (when the sum rate dominates the power consumption in the objective) the relative sum-rate gain goes up to $9 \%$. Furthermore, for very small $\alpha$, both NOMA and OMA consume the entire available power budget in order to maximize their sum rate, resulting in $E=0$. Then, NOMA first starts by consuming more power than OMA (for relatively small values of $\alpha$ when the objective is rate-driven), yielding a negative power excess $E$. Finally for larger $\alpha$ (power-efficient objectives), OMA consumes up to $33 \%$ more power than NOMA. Although for small values of $\alpha$, NOMA consumes more power than OMA, it achieves higher sum rates, which is the target goal.

It is also worth mentioning that the two metrics $G(\alpha)$ and $E(\alpha)$ exhibit opposite behavior. Specifically, the sum-rate gain 
of NOMA vs. OMA is larger for small values of $\alpha$, whereas the power excess of OMA vs. NOMA is larger for larger values of $\alpha$. This means that, when the sum rate is the critical or dominant objective (small $\alpha$ ), NOMA clearly outperforms OMA in terms of sum rate, but can consume more power. On the other hand, when the power consumption is the most critical objective (large $\alpha$ ), NOMA clearly outperforms OMA in terms of power consumption but the gain in terms of sum rate drops and might even equal zero.

Indeed, when $\alpha$ becomes too large, both OMA and NOMA achieve the same sum rate $\sum_{k} R_{k}^{\text {NOMA }}\left(\mathbf{p}^{*}(\alpha)\right)=$ $\sum_{k} R_{k}^{\mathrm{OMA}}\left(\mathbf{q}^{*}(\alpha)\right)=\sum_{k} R_{\mathrm{min}, k}$, as shown in Fig. 1(a). Since the main goal becomes minimizing the consumed power while ensuring the QoS constraints, each user is only allocated the exact power to meet its QoS. Nevertheless, as shown in Fig. 1(b), OMA requires a larger amount of power than NOMA to achieve the sum rate $\sum_{k} R_{\min , k}$.

In Fig. 2, we present the relative sum-rate gain $G$ and the relative power consumption excess $E$ as a function of the base station power budget $P_{\max }$ for five different values of $\alpha \in\{0 ; 0.2 ; 0.5 ; 1 ; 1.5\}$.

First, in Fig. 2(a) we note that NOMA always outperforms OMA in terms of sum rate for all values of $\alpha$ and power budget $P_{\text {max }}$. The largest gaps in the sum rate are achieved for small $\alpha$ i.e., when the objective reduces to maximizing the sum rate, with little or no power consumption consideration. For $\alpha>0$, the optimal solution tradeoffs between sum rate and power consumption. For large values of $\alpha$, a small and flat relative sum-rate gain can be observed: as the objective becomes more power efficient, the achieved sum rate for both NOMA and OMA approaches the sum of the minimum required user rates, irrespective from $P_{\max }$.

With respect to the power consumption, NOMA does not always outperform OMA. Indeed, in Fig. 2(b), we see that for small values of $\alpha$ and large values of $P_{\max }$, NOMA consumes more power than OMA, resulting into negatives of the excess $E$. Nevertheless, for these values of $\alpha$, the objective is sum-rate driven with little or no consideration of the power consumption. When $\alpha$ increases and the objective becomes more power efficient, it can be seen that OMA is outperformed by NOMA and may require up to $34 \%$ more power to achieve nearly the same sum rate $(\alpha=1.5)$.

\section{CONCLUSions}

In this paper, we provided a closed-form solution to the energy efficiency maximization, defined as a bi-criterion problem, in a multi-user downlink NOMA system. Our solution optimally tradeoffs between achievable sum rate and overall power consumption for any weighting factor between the two objectives. We also show that our solution is also quite useful when maximizing the ratio between the sum rate and overall power consumption.

\section{REFERENCES}

[1] M. Aldababsa, M. Toka, S. Gökçeli, G. K. Kurt, and O. Kucur, "A tutorial on nonorthogonal multiple access for $5 \mathrm{G}$ and beyond," Wireless Communications and Mobile Computing, 2018.

[2] S. R. Islam, N. Avazov, O. A. Dobre, and K.-S. Kwak, "Power-domain non-orthogonal multiple access (noma) in $5 \mathrm{~g}$ systems: Potentials and challenges," IEEE Communications Surveys \& Tutorials, vol. 19, no. 2, pp. 721-742, 2016.

[3] M. Vaezi, Z. Ding, and H. V. Poor, Multiple access techniques for $5 G$ wireless networks and beyond. Springer, 2019.

[4] Z. Chen, Z. Ding, X. Dai, and R. Zhang, "An optimization perspective of the superiority of NOMA compared to conventional OMA," IEEE Transactions on Signal Processing, vol. 65, no. 19, pp. 5191-5202, 2017.

[5] D. Zhai, R. Zhang, L. Cai, B. Li, and Y. Jiang, "Energy-efficient user scheduling and power allocation for NOMA-based wireless networks with massive IoT devices," IEEE Internet of Things Journal, vol. 5, no. 3, pp. 1857-1868, June 2018.

[6] M. Zeng, A. Yadav, O. A. Dobre, and H. V. Poor, "Energy-efficient power allocation for uplink NOMA," in 2018 IEEE Global Communications Conference (GLOBECOM). IEEE, 2018, pp. 1-6.

[7] J. Tang, J. Luo, D. So, E. Alsusa, K.-K. Wong, and N. Zhao, "Energyefficient resource allocation in SWIPT enabled NOMA systems," in 2018 IEEE Global Communications Conference (GLOBECOM). IEEE, 2018, pp. 1-7.

[8] W. U. Khan, F. Jameel, T. Ristaniemi, S. Khan, G. A. S. Sidhu, and J. Liu, "Joint spectral and energy efficiency optimization for downlink NOMA networks," IEEE Transactions on Cognitive Communications and Networking, 2019

[9] W. Shin, M. Vaezi, B. Lee, D. J. Love, J. Lee, and H. V. Poor, "Nonorthogonal multiple access in multi-cell networks: Theory, performance, and practical challenges," IEEE Communications Magazine, vol. 55, no. 10 , pp. $176-183,2017$.

[10] M. Vaezi, R. Schober, Z. Ding, and H. V. Poor, "Non-orthogonal multiple access: Common myths and critical questions," IEEE Wireless Communications, vol. 26, no. 5, pp. 174-180, 2019.

[11] Z. Yang, W. Xu, C. Pan, Y. Pan, and M. Chen, "On the optimality of power allocation for NOMA downlinks with individual QoS constraints," IEEE Communications Letters, vol. 21, no. 7, pp. 1649-1652, 2017.

[12] Y. Zhang, H.-M. Wang, T.-X. Zheng, and Q. Yang, "Energy-efficient transmission design in non-orthogonal multiple access," IEEE Transactions on Vehicular Technology, vol. 66, no. 3, pp. 2852-2857, 2016.

[13] R. Masmoudi, E. V. Belmega, I. Fijalkow, and N. Sellami, "A unifying view on energy-efficiency metrics in cognitive radio channels," in EUSIPCO, Lisbon, Portugal, Sep. 2014.

[14] S. Boyd and L. Vandenberghe, Convex Optimization. Cambridge University Press, 2004.

[15] C. Isheden, Z. Chong, E. Jorswieck, and G. Fettweis, "Framework for link-level energy efficiency optimization with informed transmitter," IEEE Trans. on Wireless Commun., vol. 11, no. 8, pp. 2946-2957, 2012. 